See discussions, stats, and author profiles for this publication at:

http://www.researchgate.net/publication/258868753

\title{
Structure and properties of nanocomposites based on PTT-block- PTMO copolymer and graphene oxide prepared by in situ polymerization
}

ARTICLE in EUROPEAN POLYMER JOURNAL · NOVEMBER 2014

Impact Factor: 3.01 · DOI: 10.1016/j.eurpolymj.2013.10.031

CITATIONS

4
READS

118

6 AUTHORS, INCLUDING:

\section{Sandra Paszkiewicz}

West Pomeranian University of Techn...

26 PUBLICATIONS 40 CITATIONS

SEE PROFILE

\section{Zdenko Spitalsky}

Slovak Academy of Sciences

58 PUBLICATIONS 1,037 CITATIONS

SEE PROFILE
Anna Szymczyk

Szczecin University of Technology

38 PUBLICATIONS 220 CITATIONS

SEE PROFILE

\section{Jaroslav Mosnacek}

Slovak Academy of Sciences

71 PUBLICATIONS 470 CITATIONS

SEE PROFILE 


\title{
Structure and properties of nanocomposites based on PTT -block-PTMO copolymer and graphene oxide prepared by in situ polymerization
}

\author{
Sandra Paszkiewicz ${ }^{\mathrm{a}, *}$, Anna Szymczyk ${ }^{\mathrm{b}}$, Zdenko Špitalský ${ }^{\mathrm{c}}$, Jaroslav Mosnáček ${ }^{\mathrm{d}}$, \\ Konrad Kwiatkowski ${ }^{\text {a }}$, Zbigniew Rosłaniec ${ }^{a}$ \\ ${ }^{a}$ West Pomeranian University of Technology, Institute of Material Science and Engineering, Piastow Av. 19, PL-70310 Szczecin, Poland \\ ${ }^{\mathrm{b}}$ West Pomeranian University of Technology, Institute of Physics, Piastow Av. 19, PL-70310 Szczecin, Poland \\ c Polymer Institute, Slovak Academy of Sciences, Dúbravská cesta 9, 84541 Bratislava 45, Slovakia \\ d Polymer Institute, Centre of Excellence FUN-MAT, Slovak Academy of Sciences, Dúbravská cesta 9, 84541 Bratislava 45, Slovakia
}

\section{A R T I C L E I N F O}

\section{Article history:}

Received 3 July 2013

Received in revised form 28 October 2013

Accepted 29 October 2013

Available online $\mathrm{xxxx}$

\section{Keywords:}

Graphene oxide

Thermoplastic elastomers

In situ polymerization

\begin{abstract}
A B S T R A C T
Poly(trimethylene terephthalate-block-tetramethylene oxide) (PTT-PTMO) copolymer/ graphene oxide nanocomposites were prepared by in situ polymerization. From the SEM and TEM images of PTT-PTMO/GO nanocomposite, it can be seen that GO sheets are clearly well-dispersed in the PTT-PTMO matrix. TEM images also showed that graphene was well exfoliated into individual sheets, suggesting that in situ polymerization is a highly efficient method for preparing nanocomposites. The influence of GO on the two-phase structure, melt viscosity and mechanical properties of PTT-PTMO block copolymer was examined by using DSC, ARES rheometer and tensile tests. The DSC results imply that the introduction of GO did not affect the glass transition temperature of PTMO-rich soft phase, melting temperature of PTT hard phase and degree of crystallinity of the nanocomposites. As the graphene oxide loading in the nanocomposites increase, the enhanced Young's modulus and yield stress was observed. The tensile strength slightly increased with the increase of GO from 0 to $0.5 \mathrm{wt} \%$ when elongation at break was higher or comparable to the value of neat PTT-PTMO copolymer.
\end{abstract}

(c) 2013 Elsevier Ltd. All rights reserved.

\section{Introduction}

Polymer composites that display a change in composition and structure over a nanometer length scale have been shown over the last 10 years to present considerable property enhancements relative to conventionally scaled composites. Layered silicates dispersed as a reinforcing phase in an engineering polymer matrix are one of the most important forms of such "hybrid organic-inorganic nanocomposites" with remarkably improved thermal, mechanical (higher modulus, increased strength), optical, barrier and physicochemical parameters [1-3].

\footnotetext{
* Corresponding author. Tel.: +48 9144940 28; fax: +48 914494356. E-mail address: spaszkiewicz@zut.edu.pl (S. Paszkiewicz).
}

Graphene, mostly in the form of graphite, has been experimentally studied for over 40 years [4-7] and measurements of transport properties in micromechanically exfoliated layers [8] of graphene grown on ( $\mathrm{SiC}$ ) [9] large area graphene grown on copper $(\mathrm{Cu})$ substrates [10], as well as a variety of studies involving the use of chemically modified graphene to make new materials [11-13]. Despite the number of methods for its synthesis, as-prepared graphene itself is not soluble and thus cannot be dispersed in water or any organic solvent. Suitably modified graphene nanosheets could display good solution chemistry with properties such as dispersability and solubility in water and organic solvents [14]. Therefore, hydrophilic and organophilic affinities for graphene nanosheets should be achievable through chemical functionalization. Many 
researchers are now focusing on derivatives of graphite, which is inexpensive and available in large quantities. A particularly popular derivatives is graphite oxide (GO), which is hydrophilic and has a larger interlayer distance than graphite. It can readily exfoliate into individual GO sheets in water and forms stable dispersions after sonication. Unlike the bulk graphene sheets - if left unprotected - will spontaneously agglomerate and even restack to form graphite, chemical functionalization or the use of dispersant generally prevents agglomeration [15-17]. The GO synthetic pathway is attractive for stabilizing individual sheets in solutions. The oxygen functional groups that exist in GO provide reactive sites for chemical modification using known carbon surface chemistry. The chemical attachment of appropriate organic groups leads to physical separation of the resultant graphene sheets but also makes it possible to directly form stable graphene dispersion during the synthetic process. The successful dispersion of graphene has enabled the use of low cost solution processing techniques to fabricate various potentially useful graphene-based materials $[18,19]$. Polymer nanocomposites with GO-derived graphene materials as filler have shown dramatic improvements in properties such as elastic modulus, tensile strength and thermal stability. Moreover, these improvements are often observed at low loadings of filler evidently due to the large interfacial area and high aspect ratio of these materials, requiring small amounts of filler to achieve percolation [20,21]. Furthermore, studies on PANIPAM/GO nanocomposite hydrogels [22] prepared by in situ polymerization have shown that introduction of GO has significant influence on the microstructure, mechanical performance and swelling properties of composite hydrogels.

Segmented block copolymers which behave as thermoplastic elastomers are composed of flexible and rigid segments. Due to structural differences, the flexible and rigid segments usually separate into two phases or domains. At room temperature, the segmented block copolymers have multi-phase structures consisting of a continuous soft phase of the flexible segments with a low glass transition temperature and a dispersed hard phase with a high melting temperature [23]. Soft phase provides extensibility, whereas hard domains play the role of physical crosslinks and act as high modulus filler. Crystallization of block copolymer micro-domains exerts tremendous influence on the morphology, properties and applications of these materials. Actually, in polyester-segmented block copolymers, the phase separation occurs mainly by crystallization [23]. Multiblock poly(ether-ester) (PEE) based on poly(butylene terephthalate) (PBT) as rigid segments and poly(tetramethylene oxide) (PTMO) as soft segments have been intensively studied [24,25]. Due to their excellent mechanical properties, like strength and elastic properties in a wide temperature range they are of special interest. The PBT-block-PTMO copolymers are available as commercial products (Elitel ${ }^{\mathrm{TM}}$, Arnitel, Hytrel ${ }^{\circledR}$, DSM, etc.). Recently a study on a novel family of polyester thermoplastic elastomers based on PTT has been conducted $[26,27]$. The influence of organoclay [28] and carbon nanotubes [29] on the structure and physical properties of PTT-block-PTMO copolymers has been previously described. These type segmented copolymers based on poly(trimethylene terephthalate) (PTT) rigid segments and polyether flexible segments possess excellent thermoplastic elastomer properties, such as a low-temperature glass transition $\left(T_{g}\right)$, a high melting point $\left(T_{m}\right)$ and a temperature independent rubbery plateau. The excellent properties of this type of polyester block copolymer make it suitable for a number of applications where mechanical strength and durability in a flexible component is required. Similar to standard Hytrel grades, PTT-based block copolymers can be an excellent choice in applications ranging from auto parts and innovative furniture design to sporting goods and filaments.

The presence of carbon nanostructures in PTT-blockPTMO matrix can influence the phase separation changing their elastic properties. The purpose of this study was to examine the effect of GO content on the phase structure and physical properties of poly(trimethylene terephthalate-block-tetramethylene oxide) (PTT-PTMO) elastomer investigated. A reinforcement by GO has been observed for PTT-PTMO/GO nanocomposites based on the increase in tensile strength and tensile modulus.

\section{Experimental section}

\subsection{Materials}

For the poly(trimethylene terephthalate-block-tetramethylene oxide) (PTT-PTMO) elastomer synthesis the following chemicals were used: dimethyl terephthalate (DMT, Sigma-Aldrich) and poly(tetramethylene oxide) glycol with molecular weight of $1000 \mathrm{~g} / \mathrm{mol}$ (PTMG, Terathane 1000, DuPont, USA) were used as received. 1,3-Propanenediol (PDO, Sigma-Aldrich) was distilled before using. Tetrabutyl orthotitaniate (TBT, Fluka) was used as catalyst in transesterification and polycondensation. Irganox 1010 (Ciba-Geigy, Switzerland) was used as antioxidant.

Graphene oxide (GO) with average particle size of $50 \mu \mathrm{m}$ was obtained from expanded graphite (SLG TECHNOLOGIES GMbH, Germany) by Brodie oxidation method reported in [14]. C1s XPS spectra of GO: sp2-C: $14.97 \%$; sp3-C: $29.47 \%$; $\mathrm{C}-\mathrm{OH}: 39.82 \%$; $\mathrm{C}=\mathrm{O}: 10.75 \%$; $\mathrm{COOH}$ : $4.40 \% ; \pi-\pi$ : $0.59 \%$. O1s XPS spectra of GO: C $-0: 90.38 \%$; $\mathrm{C}=0$ 9.62\%.

\subsection{Preparation of PTT-PTMO/GO nanocomposites}

Preparation method of nanocomposites is presented in Fig. 1. A similar procedure as previously described for PTT-PTMO/Nanofil 32 nanocomposites was used [28]. Before polymerization, a dispersion of GO sheets was prepared by dispersing the desired amount of graphite oxide in PDO through ultrasonication for 15 min using laboratory homogenizer (Sonoplus HD 2200, with frequency of $20 \mathrm{kHz}$ and $75 \%$ of power $200 \mathrm{~W}$ ) and subsequent intensive mixing for 15 min with high-speed stirrer (Ultra-Turax T25). Additionally, to improve the dispersion/exfoliation of GO in PDO an ultra-power lower sonic bath (BANDELIN electronic GMbH \& Co. KG, Sonorex Digitec, with frequency of $35 \mathrm{kHz}$ and power $140 \mathrm{~W}$ ) was applied for $20 \mathrm{~h}$. The 


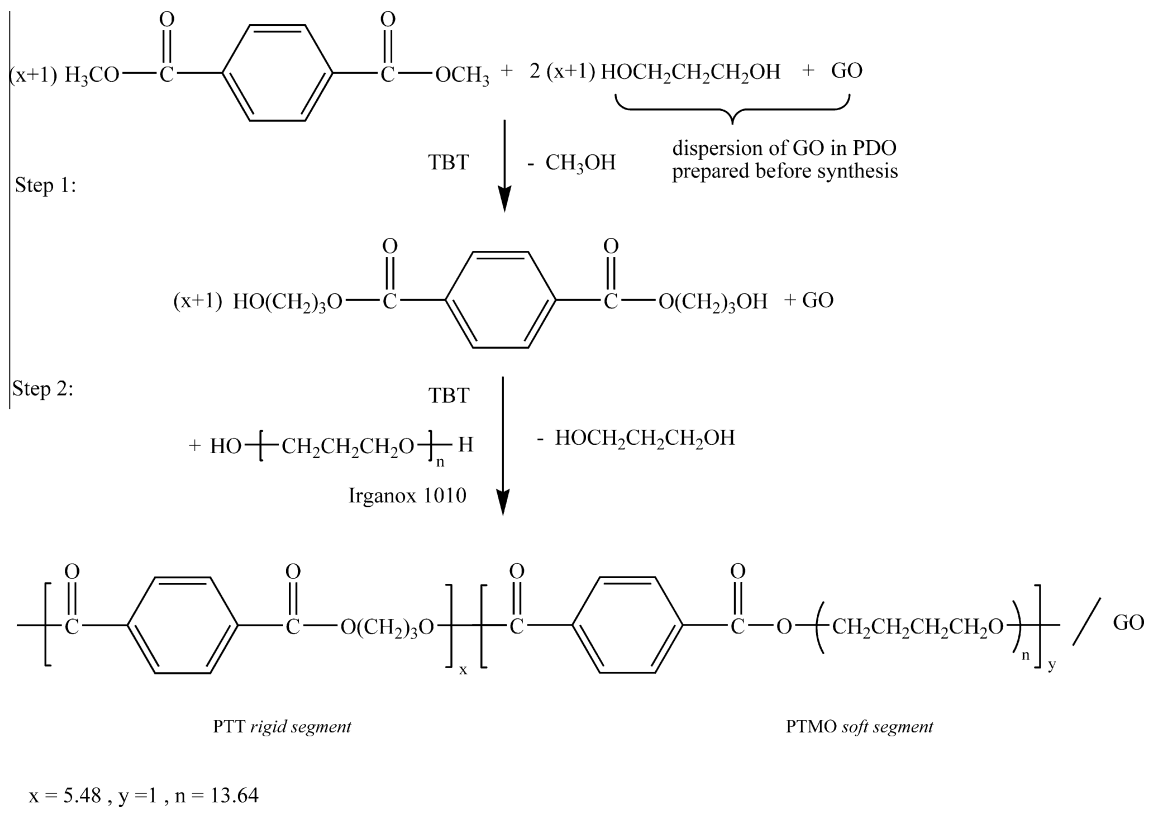

Fig. 1. Scheme of the preparation method of nanocomposites.

polymerization process was conducted in two stages. In the first stage, the dispersion of GO in PDO (3.774 mol), DMT $(0.629 \mathrm{~mol})$ and TBT catalyst was charged into $1 \mathrm{~L}$ steel reactor (Autoclave Engineers Inc, USA), where DMT was transesterified with PDO in the presence of catalyst (TBT, $0.15 \mathrm{wt} \%$ in relation to DMT) under nitrogen flow at at $165^{\circ} \mathrm{C}$ and atmospheric pressure under nitrogen flow. PDO was used in a 6-fold molar excess over DMT. During the reaction, methanol was distilled off. After $1 \mathrm{~h}$ to the reaction mixture, comprises mostly of bis-(3-hydroxypropyl) terephthalate, the PTMO (0.097 mol), Irganox 1010 ( $0.5 \mathrm{wt} \%$ of total comonomers mass) and second portion of catalyst (TBT, $0.10 \mathrm{wt} \%$ in relation to DMT) was added. Then, the temperature slowly went up to $210^{\circ} \mathrm{C}$ and stayed for half an hour to reach the endpoint of transesterification. Subsequently, the excess of PDO used in the first stage was distilled off during increasing the temperature and reducing the pressure. The second step, melt polycondensation was carried out at $250{ }^{\circ} \mathrm{C}$ under reduced pressure of $\sim 20$ Pa. During polycondensation, the torque was monitored in order to detect changes in viscosity. All syntheses were finished when melt rich the same value of viscosity at $250{ }^{\circ} \mathrm{C}$. The obtained polymer/nanocomposite was extruded from the reactor under nitrogen flow. The neat PTT-PTMO copolymer was synthesized following the same procedure, but in the absence of GO. The content of rigid PTT and soft PTMO segments was approximately the same (i.e. $50 \mathrm{wt} \%$ of each). The produced nanocomposite samples were coded as PTT-PTMO/xGO, where $\mathrm{x}$ describe the weight content of GO.

\subsection{Sample preparation}

The dumbbell shape samples for tensile tests and SAXS measurements were obtained by injection moulding using a Boy 15 (Dr. BOY GmbH\&Co., Germany) injection molding machine. The injection-molding parameters were as follows: injection pressure $55 \mathrm{MPa}$, melt temperature $225^{\circ} \mathrm{C}$, mold temperature $30^{\circ} \mathrm{C}$, holding down pressure of $20 \mathrm{MPa}$ for $15 \mathrm{~s}$ and cool time $10 \mathrm{~s}$.

\subsection{Characterization techniques}

The intrinsic viscosity $[\eta]$ of the samples was measured using Ubbelohde viscometer at $30^{\circ} \mathrm{C}$ in mixture of phenol/ 1,1,2,2-tetrachloroethane ( $60 / 40$ by weight). The polymer solution had a concentration of $0.5 \mathrm{~g} / \mathrm{dL}$. The following procedure was used to eliminate the influence of GO presence on measured $[\eta]$ values. Nanocomposite sample was dissolved in mixture of phenol/1,1,2,2-tetrachloroethane (60/40 by weight), then filtered to separate GO. The sample was precipitated by adding methanol and recovered by filtration. Finally, the precipitated solid was dried in vacuum at $50{ }^{\circ} \mathrm{C}$ for $24 \mathrm{~h}$.

Size-exclusion chromatography (SEC) in 1,1,1,3,3,3hexafluoro-2-propanol (HFIP) was performed on a Waters system equipped with a Waters 2414 refractive index detector $\left(35^{\circ} \mathrm{C}\right)$, a Waters 2707 autosampler, a Waters 1515 Isocratic HPLC pump, and a PSS PFG guard columns followed by 2 PFG-linear-XL ( $7 \mu \mathrm{m}, 8 \times 300 \mathrm{~mm}$ ) columns in series at $40{ }^{\circ} \mathrm{C}$. HFIP was used as eluent at a flow rate of $1 \mathrm{~mL} / \mathrm{min}$. The molecular weight calibration curve was obtained using of nine poly(methyl methacrylate) standards (Polymer Laboratories) of narrow polydispersity.

An ARES rheometer (Rheometric Scientific Inc., USA) was used to measure the melt viscosity of the samples. The measurements were done at temperature of $220^{\circ} \mathrm{C}$ and at frequencies in the range of $0.1-50 \mathrm{~Hz}$, in a parallel-plate fixture (diameter $=25 \mathrm{~mm}$ ) with a gap distance of $2 \mathrm{~mm}$. 
The density was measured at $23^{\circ} \mathrm{C}$ on hydrostatic balance (Radwag WPE 600C, Poland), calibrated for standards with known density.

The thermal transitions of the polymers were measured with differential scanning calorimetry (DSC, TA Instrument Q-100). The heating and cooling rate was $10^{\circ} \mathrm{C} / \mathrm{min}$ and sample size approximately of $10 \mathrm{mg}$. An indium standard was used to calibrate the temperature and the heat of fusion. The first cooling and second heating scans were used to determine the melting and crystallization peaks. The heat of fusion was determined by integration of the normalized area of melting endotherm. The glass transition temperature ( $\mathrm{Tg}$ ) for the polymer samples was taken as the midpoint of the change in heat capacity $(\Delta \mathrm{cp} / 2)$. The degree of crystallinity of the samples was calculated by the following equation: $x_{c}=\left(\Delta H_{m} / \Delta H_{m}^{o}\right)$; where $\Delta H_{m}^{o}$ $(=146 \mathrm{~J} / \mathrm{g})$ is the enthalpy change of melting for a $100 \%$ crystalline sample [26] and $\Delta H_{m}$ is derived from melting peak area on DSC thermogram.

The structure of nanocomposites was observed by scanning electron microscopy (SEM, JEOL JSM 6100). The samples were cryofractured in liquid nitrogen, and then vacuum coated with a thin gold film before the test. Transmission electron microscopy (TEM) analysis was carried out by a JEOL JEM-1200 Electron Microscope using an acceleration voltage of $80 \mathrm{kV}$. The TEM samples (thickness of about 100-150 nm) were obtained by cutting of tensile specimen's perpendicular to flow direction under cryogenic conditions using Reichert Ultracut R ultramicrotome with a diamond knife.

Tensile measurements were performed on Instron 5566 universal tensile testing frame, equipped with a $5 \mathrm{kN}$ Instron load cell, an contact optical long travel extensometer and the Bluehill 2 software, following the same procedure as that described for PTT-PTMO block copolymer [26]. Mean values and standard deviations were calculated for the various material properties: Young's modulus, yield stress, stress and strain at break, permanent set. A confidence interval for the mean was then calculated according to the ISO 2854 standard.

Small angle X-ray scattering (SAXS) measurements were performed at beam line A2 at HASYLAB (DESY, Hamburg) following the same procedure as that previously described for PTT-PTMO/Nanofil 32 nanocomposites [28]. The wavelength of the X-ray beam was $\lambda=0.15 \mathrm{~nm}$. The SAXS scattering-vectors were calibrated using a dray rattail tendon protein. Scattering patterns were collected at room temperature by a two-dimensional MAR-CCD-165 detector placed at a distance of $2443 \mathrm{~mm}$ from the sample. Exposure time was $20 \mathrm{~s}$. The raw SAXS intensity data were corrected for background scattering. The raw intensity data were corrected for background scattering. The Bragg long period $(L)$ was determined from the position of the peak in the Lorentz-corrected SAXS intensity (after background subtraction), $I(s) s^{2}$ vs. $s$.

\section{Results and discussion}

\subsection{Exfoliation of GO in PTT-b-PTMO matrix}

The maximal reinforcement effect in polymer nanocomposite can be obtained while graphene sheets are non-agglomerated, highly exfoliated and uniformly dispersed in polymer matrix, and interphase interactions ensure as strong as possible interactions between dispersed phase (reinforcement) and polymer matrix. Graphene oxide, one of the graphene derivative form, attaches many oxygen-containing hydrophilic functional groups such as - $\mathrm{COOH}$ and -OH [30-32]. Well-dispersed GO nanocomposites were fabricated trough in situ polymerization method as described in the experimental section. Thanks to many oxygen-containing functional groups on the surfaces of GO and electrostatic repulsion between the negative charge of GO sheets, GO can be very well dispersed in PDO at the level of individual sheets. The dispersion state and distribution of GO in PTT-PTMO matrix were characterized by SEM and TEM. Fig. 2 shows a typical SEM image of cross section of the PTT-PTMO/GO nanocomposites with $0.7 \mathrm{wt} \%$ loading of GO. From the SEM images of the fractured surface of PTT-PTMO/ GO nanocomposite, it can be seen that GO sheets are clearly well-dispersed in the PTT-PTMO matrix. The presence of agglomerates was not observed, only partial sections GO sheets (Fig. 2b-c) pulled out from the matrix during fracturing are visible on the nanocomposite surface. The other parts of GO were not observed because they were inside matrix. For further characterization of the PTT-PTMO/GO nanocomposite structure, we conducted TEM analysis of clipped thin tensile specimen's using ultramicrotome with a diamond knife. Good dispersion GO in PTT-PTMO matrix was confirmed by TEM analysis (Fig. 3), showing the presence of few layer GO platelets with crumpled/folded morphology. As shown in Fig. 3, TEM images show that graphene was well exfoliated into individual sheets in glycol by mechanical and ultrasonic treatment, exhibiting clearly a flake-like shape of graphene oxide, suggesting that in situ polymerization is a highly efficient method for preparing nanocomposites.

\subsection{Nanostructure of nanocomposites}

In Fig. 4 , the scattering peaks $\left(s_{\max }\right)$ associated with the electron density contrast between the alternating crystalline and amorphous layers in neat copolymer and nanocomposites are presented. For the nanocomposites the $s_{\max }$ were shifted slightly to a smaller position with the addition of GO and then maintained seemingly unchanged with further increasing GO content, indicating the value of the long period ( $L$, see Table 2 ) calculated by Bragg's law $\left(L=1 / s_{\max }\right)$ were slightly higher than that of PTT in neat copolymer. These increase of $L$ in nanocomposites could be a result of increase of amorphous layer thickness which can be caused by the restriction of mobility of polymer chain caused by existence of GO induced interfacial interactions between polymer and graphene sheets [28,29].

\subsection{Physical properties of nanocomposites}

PTT-PTMO/graphite oxide nanocomposites with 0.1 , $0.3,0.5$ and $0.7 \mathrm{wt} \%$ loading of nanofiller were prepared using in situ polymerization. Table 1 summarizes the physical properties of the obtained nanocomposites. As the GO loading level increased, the density of the obtained 

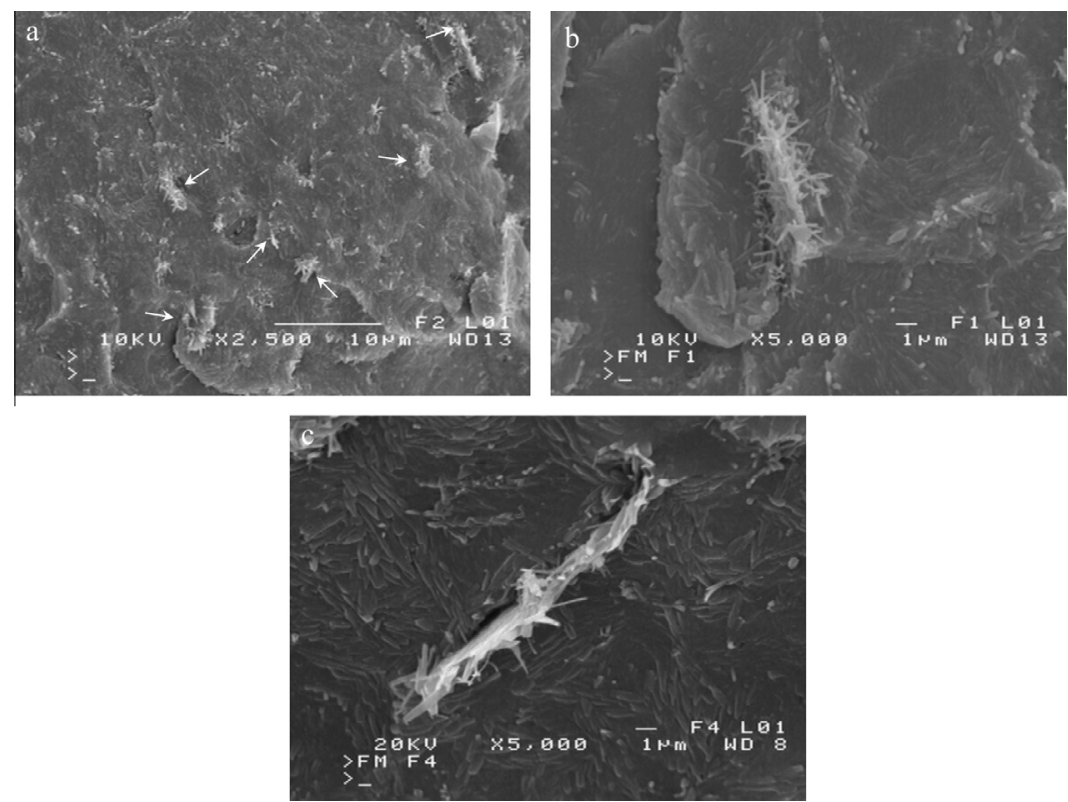

Fig. 2. SEM micrographs of the PTT-PTMO/GO (0.7 wt\%) nanocomposite: (a) $2500 \times$, (b) $5000 \times$, (c) $5000 \times$.
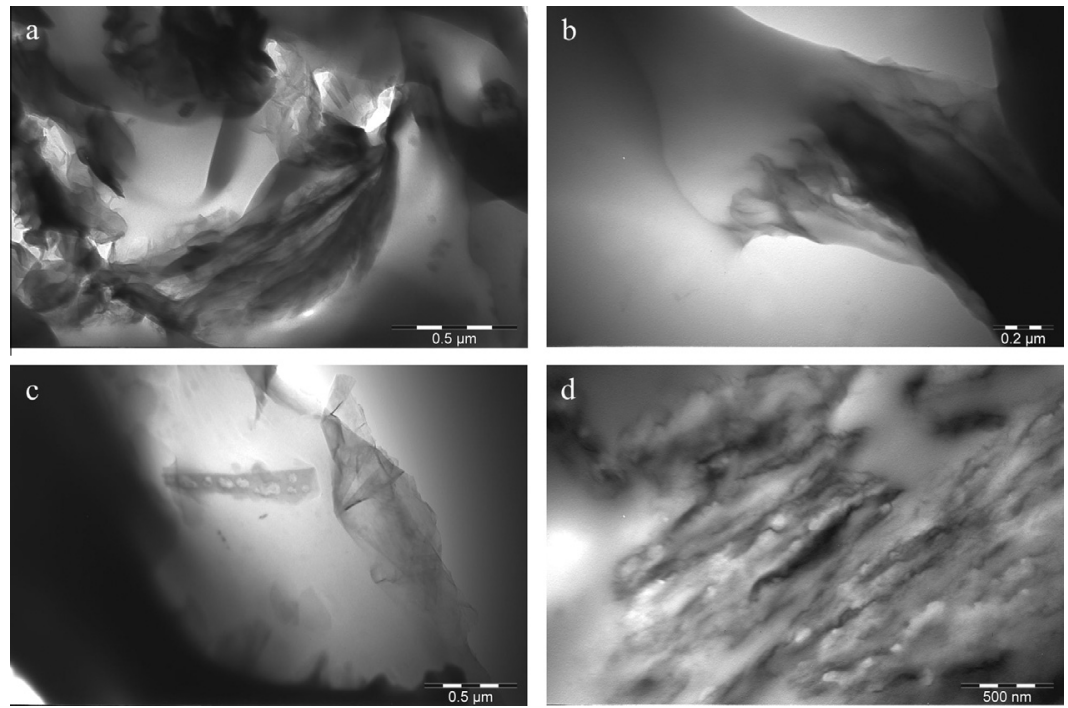

Fig. 3. TEM micrographs of the PTT-PTMO/GO nanocomposites with loading of $0.7 \mathrm{wt} \%$. (a) $100000 \times$, (b) $120000 \times$ and (c-d) $75000 \times$.

nanocomposites increase due to the presence of nanofiller with higher density.

The presence of GO in reaction mixture during synthesis of nanocomposites had influence on molecular weight of the polymer matrix in the obtained nanocomposites and their melt viscosity. The obtained values of intrinsic viscosity for nanocomposites, except the sample with $0.7 \mathrm{wt} \%$ of $\mathrm{GO}$, are very close to the neat PTT-PTMO block copolymer indicating comparable molecular weights of polymer matrices in the composites. Molecular weight of the polymer matrix in nanocomposites and its distribution determined by SEC analysis are presented in Table 1 . It can be observed that the low loading of GO does not substantially modify the PTT-PTMO copolymer number molecular weight $\left(M_{n}\right)$, which value is comparable to the $M_{n}$ of neat PTT-PTMO copolymer obtained in the absence of GO. At the higher loading of GO (0.7 wt\%), the decrease of $M_{n}$ is observed. The changes of number average molecular weight $\left(M_{n}\right)$ show a similar tendency to the changes of values of intrinsic viscosity. At the same time, molecular weight distribution $\left(M_{w} / M_{n}\right)$ of the copolymers prepared in the presence of GO by in situ polymerization increases with the concentration of GO. In many polymer nanocomposites prepared by using in situ polymerization the decrease of molecular weight and increase of $M_{w} / M_{n}$ was observed. For instance, Patole et al. $[33,34]$ have found that 


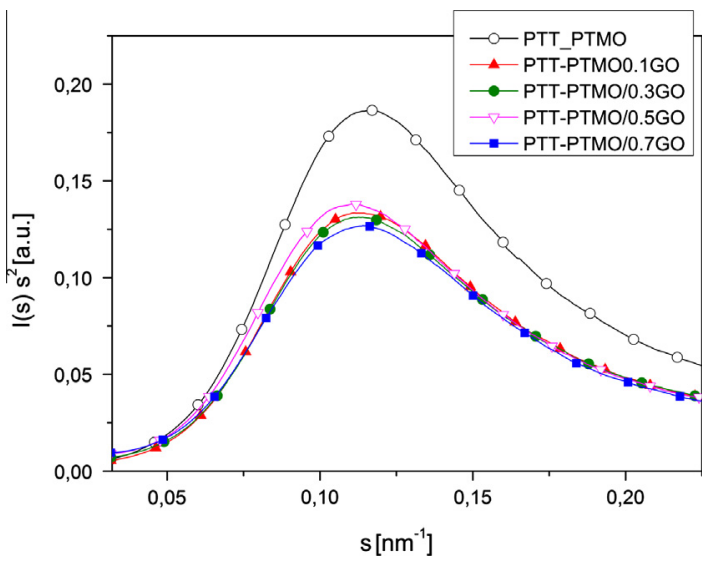

Fig. 4. The Lorentz-corrected SAXS patterns for PTT-PTMO/GO nanocomposites and neat copolymer.

the $M_{n}$ of polymer matrix decreases and $M_{w} / M_{n}$ increases with increasing nanofiller content (graphene/carbon nanotubes, clay) in polystyrene nanocomposites.

It is known that rheological properties of nanocomposites containing layered nanofillers are related to the degree of exfoliation of nanofiller in polymer matrix and also to the level of interfacial interaction between the nanofiller surface and polymer chains [35]. Usually, in nanocomposites the increase of melt viscosity is observed with increasing of nanofiller content due to nanofiller-polymer interactions. The dependence of melt viscosity of neat PTT-PTMO copolymer and nanocomposites measured at different frequencies is presented in Fig. 5. With increase of frequency, the melt viscosity of neat PTT-PTMO copolymer and nanocomposites decreases. In comparison to the neat PTT-PTMO copolymer, the melt viscosity of nanocomposites at low GO loading ( $<0.5 \mathrm{wt} \%$ ) is slightly reduced, and then increases at the higher GO loading. It is not clear what mechanism causes the reduction of melt viscosity in the nanocomposites with low GO loading. One possibility is acting of GO sheets as plasticizer, increasing the free volume and therefore decreasing the melt viscosity [36]. Whereas at higher GO loading, the polymer-GO and GOGO interactions are predominate and causes increase the melt bulk viscosity. The possible mechanisms of selective adsorption [37], excluded free volume [38] and ball-bearing effect [39] are also reported in literature to explain the decrease in melt viscosity when nanofillers are introduced to the polymer matrix. Another possible explanation for observed melt viscosity changes with GO loading can result from lubricant behaviour of graphite [40]. The graphene layers coupled by week van der Waals-like bonds

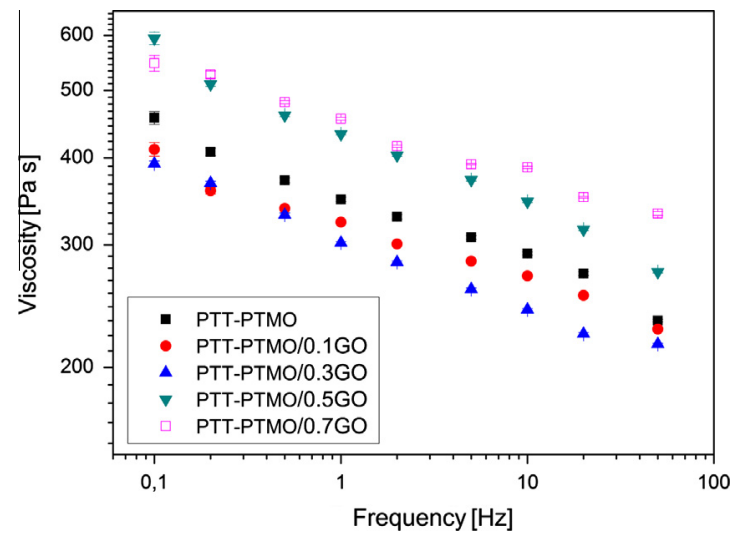

Fig. 5. Met viscosity versus frequency for neat PTT-PTMO copolymer and PTT-PTMO/GO nanocomposites at temperature of $220^{\circ} \mathrm{C}$.

can easily slide past each other. At low GO loading, few layer graphene in the studied composites can act as lubricant reducing melt viscosity.

\subsection{Thermal properties of nanocomposites}

The neat PTT-PTMO block copolymer has two phase morphology: a PTT crystalline phase and PTMO-rich amorphous phase. It was found in many semicrystalline systems that the addition of nano-additives have affected the crystalline structure and crystallization rate [29,41,42]. DSC analysis was performed in order to asses possible changes in crystalline structure and degree of crystallinity of the matrix. Fig. 6 shows heating and cooling DSC traces of neat PTT-PTMO block copolymer and PTT-PTMO/GO nanocomposites. From the obtained cooling and heating curves (Fig. 6) the thermal parameters such as glass transition temperature $\left(T_{g}\right)$, melting temperature $\left(T_{m}\right)$, crystallization temperature $\left(T_{c}\right)$, melting enthalpy, crystallization enthalpy, and degree of crystallinity (Table 2) were determined. As shown in Fig. 6, $T_{g}$ corresponding to the soft PTMO-rich amorphous phase of the prepared PTT-PTMO block copolymer is about $-67^{\circ} \mathrm{C}$. The $T_{g}$ of nanocomposites remained unaffected by incorporation of GO. This can indicate that the chain mobility of polymer chain in soft phase was not affected by the presence of GO. Regarding the effect of the GO on the $T_{m}$, slight increase $\left(2-6{ }^{\circ} \mathrm{C}\right)$ was observed, while the degrees of crystallinity of the prepared nanocomposites were comparable to the neat PTT-PTMO thermoplastic elastomer. At low loading of GO in PTT-PTMO matrix, the values of $T_{c}$ of nanocomposites were close to the $T_{c}$ of neat PTT-PTMO. These could imply that addition

Table 1

Physical properties of PTT-PTMO/GO nanocomposites.

\begin{tabular}{lllllll}
\hline Sample & $\mathrm{GO}(\mathrm{wt} \%)$ & {$[\eta](\mathrm{dl} / \mathrm{g})$} & $M_{n} \times 10^{3}(\mathrm{~g} / \mathrm{mol})$ & $M_{w} \times 10^{3}(\mathrm{~g} / \mathrm{mol})$ & $M_{w} / M_{n}$ & $d\left(\mathrm{~g} / \mathrm{cm}^{3}\right)$ \\
\hline PTT-PTMO & 0 & 1.30 & 41.7 & 88.0 & 2.11 & 1.172 \\
PTT-PTMO/0.1GO & 0.1 & 1.27 & - & 95.1 & - & 1.174 \\
PTT-PTMO/0.3GO & 0.3 & $1.28 ; 1.30^{\mathrm{F}}$ & 42.1 & 95.7 & 1.176 \\
PTT-PTMO/0.5GO & 0.5 & $1.29 ; 1.28^{\mathrm{F}}$ & 38.9 & 92.1 & 2.46 & 1.177 \\
PTT-PTMO/0.7GO & 0.7 & $1.26 ; 1.24^{\mathrm{F}}$ & 36.2 & 2.54 & 1.179 \\
\hline
\end{tabular}

$[\eta]$ - intrinsic viscosity; $d$ - density at $23^{\circ} \mathrm{C} ; F$ - measured after filtration of GO; $M_{n}$ - number average molar mass; $M_{w}$ - weight average molar mass; $M_{w} / M_{n}$ - dispersity. 

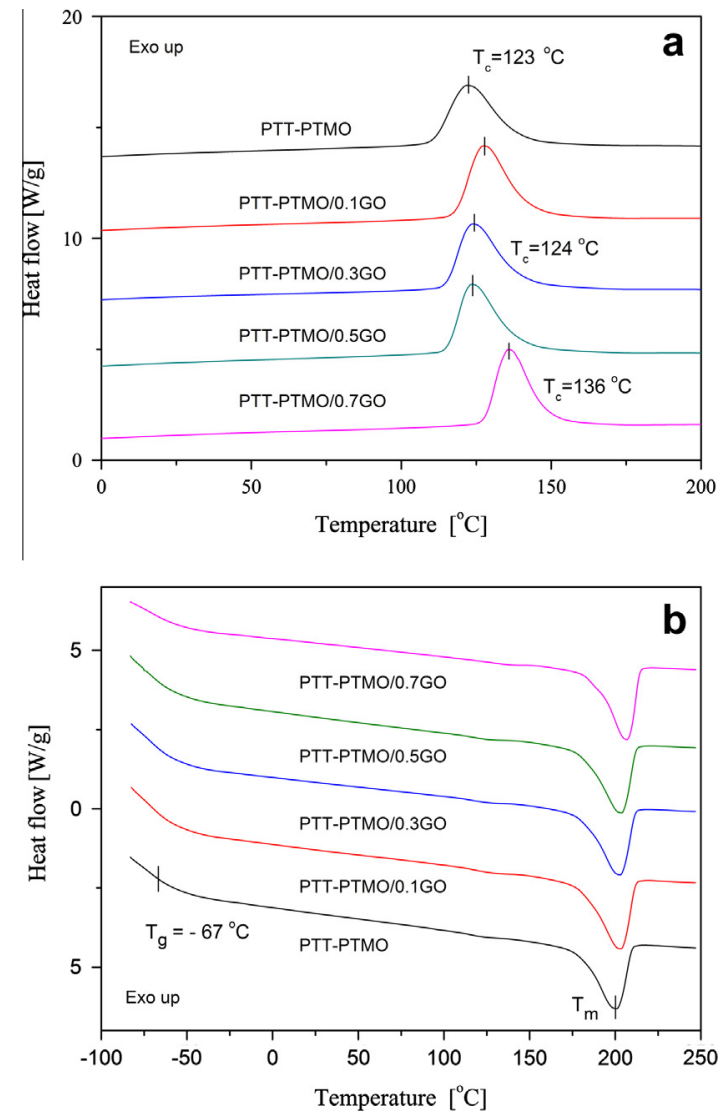

Fig. 6. DSC thermograms obtained during cooling (a) and heating (b) for PTT-PTMO/GO nanocomposites.

of GO has no influence on the crystallization rate of PTT hard domains. At higher wt\% GO loading (sample PTTPTMO/0.7GO), shift of $T_{c}$ to higher temperatures $\left(\Delta T_{c}=13^{\circ} \mathrm{C}\right)$ was observed. However these increase of crystallization rate could be rather caused by increase of the copolymer polydisperisty $\left(M_{w} / M_{n}\right)$ (Table 1) than nucleating effect of the additives.

\subsection{Tensile properties of nanocomposites}

Table 3 summarizes the mechanical properties of neat PTT-PTMO block copolymer and its graphene oxide composites. The representative stress-strain curves from tensile tests for nanocomposites are presented in Figs. 7 and 8. Error bars in Table 3 represent the 95\% confidence interval of a mean value of each determined tensile property. Analysis of the Young's modulus and their uncertainty have shown that modulus of nanocomposites was enhanced at loading of GO above $0.3 \mathrm{wt} \%$. The tensile strength slightly increased (Fig. 7) with the increase of GO from 0 to $0.5 \mathrm{wt} \%$ when elongation at break was higher or comparable to the value of neat PTT-PTMO copolymer. For the $0.7 \mathrm{wt} \% \mathrm{GO}$ loaded nanocomposite, the value of tensile strength is on the same level as for nanocomposite with 0.5 wt\% GO but of strain at break was slightly reduced. The yield stress increases slowly with increasing GO content but strain at yield slightly increases at low GO loading then decreases at the highest ( 0.7 wt\%) GO content. Similar results were obtained by Durmus et al. [43] for polyethylene (LLDPE)/clay nanocomposites.

The strengthening of composite material is a result of two major contributions, direct strengthening and indirect strengthening [44]. The direct strengthening is based on the load transfer from matrix to the higher stiffness reinforcement particles. Indirect strengthening results from the changes in the matrix microstructure that take place due to presence of reinforcement particles. In semicrystalline nanocomposites the effect of nanofiller on the stress at break values depends on the interfacial interactions between polymer and graphene sheets. Strong interfacial interaction causes the enhancement in stress at break. It was already reported $[45,46]$ that solid particles might nucleate the polymer crystallization, increasing its crystallinity, decreasing crystallite thicknesses, and influencing the orientation of the lamellae in the crystallites. This can influence the mechanical properties of the nanocomposites. Calorimetric studies for obtained PTT-PTMO/GO nanocomposites (Table 2) showed small differences in the polymer melt enthalpy (only $2.8 \mathrm{~J} / \mathrm{g}$ increase for PTTPTMO/0.7 wt\% GO) and negligible increase in degree of crystallinity. Therefore, the observed here improvement in the tensile properties at low GO loading cannot be due to a change in crystallinity and is more likely caused by the presence of GO sheets next to PTT hard domains dispersed in PTMO-rich soft phase.

The elastic deformability and reversibility of the nanocomposites and neat PTT-PTMO block copolymer were also studied during cyclic tensile test (Fig. 8). Determined values of permanent set (PS) in tension direction resultant from maximum attained stain (100\% and $200 \%$ ) for nanocomposites are presented in Table 2. As can be expected, addition of GO reduces the elasticity of the PTT-PTMO elastomer at higher loading of GO (0.5-0.7 wt\%) which is manifested by increasing of permanent set.

Table 2

Thermal properties and long period of PTT-PTMO/GO nanocomposites.

\begin{tabular}{|c|c|c|c|c|c|c|c|}
\hline Sample & $T_{g}\left({ }^{\circ} \mathrm{C}\right)$ & $T_{m}\left({ }^{\circ} \mathrm{C}\right)$ & $\Delta H_{m}(\mathrm{~J} / \mathrm{g})$ & $T_{c}\left({ }^{\circ} \mathrm{C}\right)$ & $\Delta H_{c}(\mathrm{~J} / \mathrm{g})$ & $x_{c}(\%)$ & $L(\mathrm{~nm})$ \\
\hline PTT-PTMO & -67 & 201 & 30.3 & 123 & 30.6 & 20.7 & 8.58 \\
\hline PTT-PTMO/0.1GO & -67 & 203 & 31.1 & 127 & 31.4 & 21.3 & 8.85 \\
\hline PTT-PTMO/0.3GO & -67 & 203 & 31.4 & 124 & 30.7 & 21.5 & 8.88 \\
\hline PTT-PTMO/0.5GO & -67 & 204 & 31.5 & 123 & 32.0 & 21.6 & 9.02 \\
\hline PTT-PTMO/0.7GO & -67 & 207 & 33.1 & 136 & 33.5 & 22.7 & 8.75 \\
\hline
\end{tabular}

$T_{g}$ - glass transition temperature of soft phase; $T_{m}$ - melting temperature of polyester crystalline phase; $T_{c}-$ crystallizing temperature of polyester crystalline phase; $\Delta H_{m}, \Delta H_{c}$ - enthalpy of melting and crystallization of polyester crystals, respectively; $x_{c}-$ degree of crystallinity; $L$ - long period. 
Table 3

Tensile properties of PTT-PTMO/GO nanocomposites.

\begin{tabular}{|c|c|c|c|c|c|c|c|}
\hline Sample & $E(\mathrm{MPa})$ & $\sigma_{y}(\mathrm{MPa})$ & $\varepsilon_{y}(\%)$ & $\sigma_{b}(\mathrm{MPa})$ & $\varepsilon_{b}(\%)$ & PS(100) (\%) & $\mathrm{PS}(200)(\%)$ \\
\hline PTT-PTMO & $118.4 \pm 3.4$ & $11.6 \pm 0.2$ & $36.4 \pm 1.2$ & $23.6 \pm 0.7$ & $632 \pm 22$ & $32 \pm 4$ & $89 \pm 3$ \\
\hline PTT-PTMO/0.1GO & $120.5 \pm 2.3$ & $12.1 \pm 0.3$ & $36.2 \pm 0.4$ & $24.3 \pm 0.4$ & $631 \pm 17$ & $32 \pm 2$ & $91 \pm 3$ \\
\hline PTT-PTMO/0.3GO & $121.5 \pm 3.1$ & $12.5 \pm 0.4$ & $37.4 \pm 0.5$ & $24.9 \pm 0.6$ & $644 \pm 24$ & $33 \pm 2$ & $92 \pm 4$ \\
\hline PTT-PTMO/0.5GO & $124.3 \pm 5.2$ & $12.6 \pm 0.2$ & $37.9 \pm 1.1$ & $25.5 \pm 0.7$ & $670 \pm 31$ & $35 \pm 2$ & $94 \pm 6$ \\
\hline PTT-PTMO/0.7GO & $133.5 \pm 3.2$ & $13.2 \pm 0.2$ & $33.3 \pm 0.8$ & $25.4 \pm 1.2$ & $620 \pm 26$ & $39 \pm 3$ & $98 \pm 5$ \\
\hline
\end{tabular}

$E$ - Young's modulus, $\sigma_{y}, \varepsilon_{y}$ - yield stress and strain respectively; $\sigma_{b}, \varepsilon_{b}-$ stress and strain at break respectively; PS(100), PS(200) - permanent set was taken as the strain at which zero load was measured on the first unloading cycle to $100 \%$ and $200 \%$ strain, respectively. The error bars represent the $95 \%$ confidence interval.

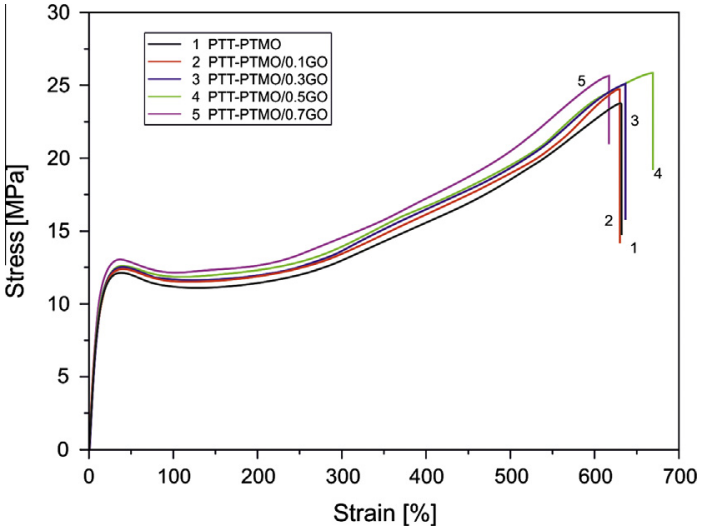

Fig. 7. Representative stress-strain curves for nanocomposites and neat PTT-PTMO copolymer.

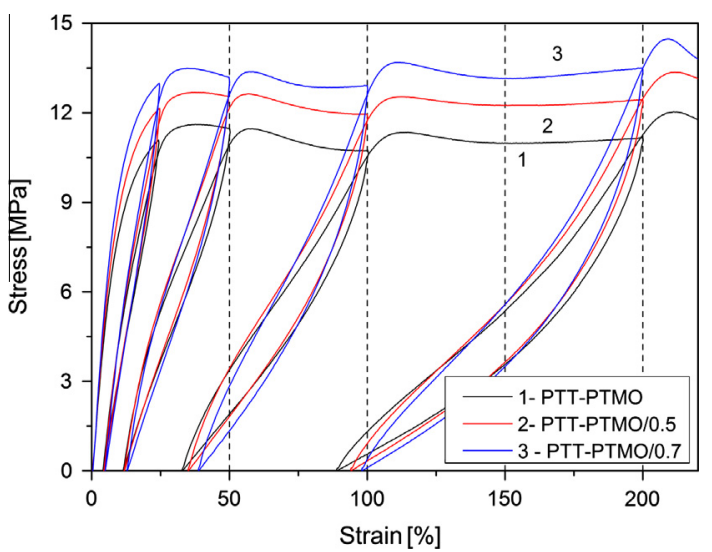

Fig. 8. The stress versus strain in cyclic tensile tests with various maximum strains $\left(\varepsilon_{\max }=25 \%, 50 \%, 100 \%, 200 \%\right)$. A representative example of the step-cycle test for neat PTT-PTMO copolymer and PTT/PTMO nanocomposites with 0.5 and $0.7 \mathrm{wt} \%$ of GO.

\section{Conclusions}

Graphene oxide filled PTT-PTMO nanocomposites were manufactured in this study. The oxygen functional groups that exist in GO provide reactive sites for chemical modification using known carbon surface chemistry. The molecular weight distribution of PTT-PTMO copolymer in the nanocomposite increases with increase of GO loading. TEM and SEM micrographs verified that the dispersion of the GO into the PTT-PTMO matrix was rather homogeneous and highly exfoliated graphene sheets are present in polymer matrix suggesting that in situ polymerization is a highly efficient method for preparing nanocomposites with low loading of GO. DSC analysis of composites containing increasing concentration of GO indicates there was no significant change on the glass transition temperature $\left(T_{g}\right)$ of soft amorphous phase and only slight increase of melting temperature $\left(T_{m}\right)\left(2-6{ }^{\circ} \mathrm{C}\right)$, while the degrees of crystallinity of the prepared nanocomposites were comparable to the neat PTT-PTMO thermoplastic elastomer. Moreover, the long period of PTT crystals increased with increase of GO concentration in PTT-PTMO matrix. The obtained nanocomposites with GO as filler have shown improvements in properties such as tensile modulus and yield stress. At higher GO loading (0.5-0.7 wt\%), the values permanent set of nanocomposites were slightly higher in comparison to the neat PTT-PMO block copolymer. Moreover, these improvements were observed at low loadings of filler evidently due to the large interfacial area and high aspect ratio of these materials.

\section{Acknowledgement}

The authors thank the financial support from MNT ERANET 2012 project (APGRAPHEL) for sponsored the study. The experiments performed at A2 in HASYLAB (DESY) were done using the beamtime of the proposal II-20080143EC.

\section{References}

[1] Ajayan PM, Schadler LS, Braun PV. Nanocomposite Science and Technology. Verlag: Wiley-VCH; 2003.

[2] Rao YQ, Pochan JM. Mechanics of polymer-clay nanocomposites. Macromolecules 2007;40:290-6.

[3] Sabu T, Ranimol S. Rubber Nanocomposites Preparation, Properties and Applications. John Wiley \& (Sons) Eds; 2010.

[4] Boehm HP, Clauss A, Fischer GO, Hofmann U. Das adsorptionsverhalten sehr dunner Kohlenstoff-Folien. Anorg Allg Chem 1962;316:119-27.

[5] Boehm HP, Setton R, Stumpp E. Nomenclature and terminology of graphite intercalation compounds. Carbon 1986;24:241-5.

[6] Lu XK, Huang H, Nemchuk N, Ruoff RS. Patterning of highly oriented pyrolytic graphite by oxygen plasma etching. Appl Phys Lett 1999;75:193-6.

[7] Van Bommel J, Crombeen JE, Vantooren A. LEED and Auger electron observations of SiC (0001) surface. Surf Sci 1975;48:463-72.

[8] Novoselov KS, Geim AK, Morozov SV, Jiang D, Zhang Y, Dubonos SV, et al. Electric field effect in atomically thin carbon films. Science 2004;306:666-9.

[9] Berger C, Song ZM, Li TB, Li XB, Ogbazghi AY, Feng R, et al. Ultrathin epitaxial graphite: 2D electron gas properties and a route toward graphene-based nanoelectronics. J Phys Chem B 2004;108:19912-6. 
[10] Li XS, Cai WW, An JH, Kim S, Nah J, Yang DX, et al. Large-area synthesis of high-quality and uniform graphene films on copper foils. Science 2009;324:1312-4.

[11] Stankovich S, Dikin DA, Dommett GHB, Kohlhaas KM, Zimney EJ, Stach EA, et al. Graphene-based composites materials. Nature 2006;442:282-6.

[12] Dikin DA, Stankovich S, Zimney EJ, Piner RD, Dommett GHB, Evmenenko G, et al. Preparation and characterization of graphene oxide paper. Nature 2007;448:457-60.

[13] Park S, Ruoff RS. Chemical methods for the production of graphenes. Nat Nanotechnol 2009;4:217-24.

[14] Špitalský Z, Danko M, Mosnáček J. Preparation of functionalized graphene sheets. Curr Org Chem 2011;15:1133-50.

[15] Xu Y, Bai H, Lu G, Li C, She G. Flexible graphene films via the filtration of water-soluble noncovalent functionalized graphene sheets. J Am Chem Soc 2008;130:5856-7.

[16] Stankovich S, Piner RD, Chen X, Wu N, Nyugen ST, Ruoff RS. Stable aqueous dispersions of graphitic nanoplatelets via the reduction of exfoliated graphite oxide in the presence of poly(sodium 4styrenesulfonate). J Mater Chem 2006;16:155-8.

[17] Niyogi S, Bekyarowa E, Itkis ME, Williams JL, Hamon MA, Haddon RC. Solution properties of graphite and graphene. J Am Chem Soc 2006;128:7720-1.

[18] Li D, Kaner RB. Graphene-based materials. Science 2008;320:1170-1.

[19] Huang X, Yin Z, Wu S, Qi X, He Q, Qichun Zhang Q, et al. GrapheneBased Materials: synthesis, characterization, properties and applications. Small 2011;7:1876-902.

[20] Ramanathan T, Abdala AA, Stankovich S, Dikin DA, Herrera-Alonso M, Piner RD, et al. Functionalized graphene sheets for polymer nanocomposites. Nat Nanotechnol 2008;3:327-31.

[21] Steurer P, Wissert R, Thomann R, Mulhaupt R. Functionalized graphenes and thermoplastic nanocomposites based upon expanded graphite oxide. Macromol Rapid Commun 2009;30:316-27.

[22] Ma X, Li Y, Wang W, Ji Q, Xia Y. Temperature-sensitive poly(Nisopropylacrylamide)/graphene oxide nanocomposite hydrogels by in situ polymerization with improved swelling capability and mechanical behaviour. Eur Polym J 2013;49:389-96.

[23] Schuur M, Gaymans R. Influence of morphology on the properties of segmented block copolymers. Polymer 2007;48:1998-2006.

[24] Gabrielse W, Soliman M, Dijkstra K. Microstructure and phase behavior of block copoly(ether-ester) thermoplastic elastomers. Macromolecules 2001;34:1685-93.

[25] Schmalz H, Abetz V, Lange R, Soliman M. New thermoplastic elastomers by incorporation of nonpolar soft segment in PBTbased copolymers. Macromolecules 2001;34:795-800.

[26] Szymczyk A, Senderek E, Nastalczyk J, Roslaniec Z. New multiblock poly(ether-ester)s based on poly(trimetylene terephthalate) as rigid segment. Eur Polym J 2008;44:436-43.

[27] Szymczyk A, Nastalczyk J, Sablong RJ, Roslaniec Z. The influence soft segment length on structure and properties of poly(trimetylene terephthalate)-block-poly(tetramethylene oxide) segmented random copolymers. Polym Adv Technol 2011;21:72-83.

[28] Szymczyk A, Paszkiewicz S, Roslaniec Z. Influence of intercalated organoclay on the phase structure and physical properties of PTTPTMO block copolymers. Polym Bull 2013;70:1575-90.
[29] Szymczyk A. Poly(trimethylene terephthalate-block-tetramethylene oxide) elastomer/single-walled carbon nanotubes nanocomposites: synthesis, structure, and properties. J Appl Polym Sci 2012;126:796-807.

[30] Stankovich S, Dikin DA, Piner RD, Kohlhaas KA, Kleinhammes A, Jia Y, et al. Synthesis of graphene-based nanosheets via chemical reduction of exfoliated graphite oxide. Carbon 2007;45:1558-65.

[31] Schniepp HC, Li JL, McAllister MJ, Sai H, Herrera-Alonso M, Adamson $\mathrm{DH}$, et al. Functionalized single graphene sheets derived from splitting graphite oxide. J Phys Chem B 2006;110:8535-9.

[32] Si YC, Samulski ET. Synthesis of water soluble graphene. Nano Lett 2008;8:1679-82.

[33] Patole AS, Jung S-Y, Yoo J-B, An J-H, Kim T-H. Self assembled graphene/carbon nanotube/polystyrene hybrid nanocomposite by in situ microemulsion polymerization. Eur Polym J 2012;48:252-9.

[34] Yalcinkaya SE, Yildiz N, Sacak M, Calimli A. Preparation of polystyrene/ montmorillonite nanocomposites: optimization by response surface methodology (RSM). Turk J Chem 2010;34:581-92.

[35] Ray SS. Rheology of polymer/layered silicate nanocomposites. J Ind Eng Chem 2006;12:811-42.

[36] Roberts C, Cosgrove T, Schmidt RG, Gordon GV. Diffusion of poly(dimethylsiloxane) mixtures with silicate nanoparticles. Macromolecules 2001;34:538-43.

[37] Jain S, Goossens JGP, Peters GWM, Van Duin M, Lemstra PJ. Strong decrease in viscosity of nanoparticle-filled polymer melts through selective adsorption. Soft Matter 2008;4:1848-54.

[38] Mackay ME, Dao TT, Tuteja A, Ho DL, Van Horn B, Kim HC, et al. Nanoscale effects leading to non-Einstein-like decrease in viscosity. Nat Mater 2003;2:762-6.

[39] Xie XL, Liu QX, Li RKY, Zhou XP, Zhang QX, Yu ZZ, et al. Rheological and mechanical properties of $\mathrm{PVC} / \mathrm{CaCO}_{3}$ nanocomposites prepared by in situ polymerization. Polymer 2004:45:6665-73.

[40] You Y-L, Li D-X, Deng X, Li W-L, Xie Y. Effect of solid lubricants on tribological behavior of glass fiber reinforced polyamide 6. Polym Comp 2013;34:1783-93.

[41] Müller AJ, Arnal ML, Trujillo M, Lorenzo AT. Super-nucleation in nanocomposites and confinement effects on the crystallizable components within block copolymers, miktoarm star copolymers and nanocomposites. Eur Polym J 2011;47:614-29.

[42] Wu Z, Zhou C, Zhou N. The nucleating effect of montmorillonite on crystallization of nylon 1212/montmorillonite nanocomposites. Polym Test 2002;21:479-83.

[43] Durmus A, Kasgöz A, Macosko CW. Mechanical properties of linear low-density polyethylene (LLDPE)/clay nanocomposites: estimation of aspect ratio and interfacial strength by composite models. J Macromol Sci Part B: Phys 2008;47:608-19.

[44] Ganesh VV, Chawla N. Effect of particle orientation anisotropy on the tensile behavior of metal matrix composites: experiments and microstructure-based simulation. Mater Sci Eng A 2005;391:342-53.

[45] Pukánszky B, Belina K, Rockenbauer A, Maurer FHJ. Effect of nucleation, filler anisotropy and orientation on the properties of PP composites. Composites 1994;25:205-14.

[46] Bartczak Z, Argon AS, Cohen RE, Kowalewski T. The morphology and orientation of polyethylene in films of sub-micron thickness crystallized in contact with calcite and rubber substrates. Polymer 1999;40:2367-80. 